\title{
Effects of a Brief Health Education Intervention on AIDS among Young Chinese Adults in Hong Kong
}

\author{
Abu Saleh Mohammad Abdullah, ${ }^{*, a}$ Richard Fielding, ${ }^{a}$ Anthony Johnson Hedley, ${ }^{a}$ \\ and Sunita Mahtani Stewart ${ }^{b}$
}

${ }^{a}$ Department of Community Medicine, the University of Hong Kong, 5/F William M.W. Mong Block, Faculty of Medicine Building, 21 Sassoon Road, Hong Kong and ${ }^{b}$ Department of Psychiatry, the University of Texas Southwestern Medical Center at Dallas, 5323 Harry Hines Boulevard, Dallas, Texas 75390, U.S.A.

(Received June 11, 2004; Accepted December 7, 2004)

\begin{abstract}
To examine the effects of an HIV/AIDS educational intervention among young Chinese adults in Hong Kong, we carried out a study among a group of 118 young subjects (most aged 18-25 years). Subjects were recruited from a service force and were assigned to intervention and control groups. Respondents in the intervention group attended one 90-min educational session. At baseline there was no significant difference in different variables among participants in the study. At follow-up four months later, participants in the HIV/AIDS intervention group had increased knowledge of AIDS, more positive attitudes towards AIDS prevention, held higher perception of sexually transmitted disease (STD) risk, showed higher intention to use condoms with regular and irregular partners and talked more with friends about safer sex. Respondents in the control group had more positive attitudes towards AIDS prevention and held higher perception of illness risk with no other significant changes. This first intervention study among young Chinese adults in Hong Kong identified the usefulness and limitations of an HIV/AIDS educational program. The effectiveness and limitation of the current intervention serve as an initial attempt in conducting similar studies in the future targeting the Chinese youth.
\end{abstract}

Key words — AIDS/HIV, Chinese youth, education

\section{INTRODUCTION}

Acquired immune deficiency syndrome (AIDS) and the human immunodeficiency virus (HIV) has created a major health crisis accounting for between 34-46 million infections worldwide by December 2003. ${ }^{1)}$ Young adults (15-24 years old) are the most vulnerable group for HIV infection, accounting for almost half of new infections with HIV, ${ }^{2}$ mainly because of their inexperience with sexual activities. ${ }^{3)}$ In countries with high HIV prevalence, young adults are at increased risk of infection as soon as they start having sex, because their partners are often already infected. ${ }^{2}$ Furthermore, high-risk behaviours, such as substance use, multiple partners and inconsistent condom use ${ }^{3)}$ are common among young people. To this end, making information and preventive services

*To whom correspondence should be addressed: Department of Community Medicine, the University of Hong Kong, 5/F Academic Block, Faculty of Medicine Building, 21 Sassoon Road, Hong Kong. Tel.: +852-2819-9199, -2819, -9280; Fax: +8522855-9528; E-mail: asm.abdullah@graduate.hku.hk available to young people and equipping them with the skills to negotiate with partners for safer sex is important to minimise infection risk.

The Hong Kong Special Administrative Region (HKSAR) is an economically advanced post-industrial conurbation adjacent to southern mainland China. With a total area of 1095 square kilometers, it is inhabited by 6.8 million, principally ethnic Chinese people, approximately $14 \%$ of whom are aged 15-24 years. ${ }^{4)}$ Although only $11 \%$ of the cumulative total of 1146 HIV cases in Hong Kong by the end of December 1998 were in this age group, ${ }^{5)}$ the high sexual risk-taking behaviours such as premarital sex, multiple sex partners and unprotected sexual intercourse are common among them. ${ }^{6}$ Furthermore $42 \%$ of the 3367 registered substance use cases in 1998 involved those under the age of $21,{ }^{6}$ reflecting young peoples' vulnerability to HIV infection.

Although the prevalence of HIV is not high among young adults at the moment, their high-risk behaviours may make them vulnerable to HIV infection. Studies conducted by the Family Planning Association of Hong Kong ${ }^{7,8)}$ have reported an in- 
crease in risky sexual behaviour among youths. It is therefore imperative for young people to know what risks exist and how to avoid them if they are to avoid contracting HIV.9) Various studies ${ }^{7,8,10)}$ have been carried out among young people in Hong Kong to estimate HIV infection risk but none reported any educational interventions. Moreover, discussion of sexual issues within the family is not common in Hong Kong ${ }^{11)}$ and sex education in schools is limited to developing teaching kits for primary and secondary school students. In addition, the period following school leaves young adults without any educational support for sexual health. Therefore AIDS educational intervention targeting young people is an urgent issue in Hong Kong. In this study, we have selected youth from the police training school who were conveniently available and the socio-demographic background of whom are similar to those of the average Hong Kong youths. The main aim of the intervention designed in this study is to increase knowledge of and attitudes towards AIDS, a positive change in behavioural intentions towards safer sexual practice and in perception of risks.

\section{MATERIALS AND METHODS}

Design — A quasi-experimental design with preand post-intervention measures was employed to evaluate the effectiveness of the intervention amongst the two groups of adolescents: an intervention group, which received the intervention, and a comparison group, which did not receive intervention.

Setting and Subjects — Subjects were recruited in February 1997 from the Police Training School (PTS) recruits who joined the institute for a sixmonth training. Verbal consent was obtained from the subjects before their participation in the study.

There were a total of 154 recruits (136 males and 18 females) enrolled in the PTS at the time of the program and we have recruited only the males in the study. Of all the 136 males, about half (72) were assigned to the intervention and 64 to comparison groups. Eighteen respondents ( 5 from the intervention group and 13 from the comparison group) left the institute before follow-up, leaving 67 and 51 in the intervention and comparison group, respectively. Assignments to intervention or comparisongroup were based on the basis of their living and class schedule arrangements, blinded to other demographic factors. Those who were included in the intervention group were not living in the same room, did not attend a joint training session and were not from the same class as those in the comparison group. This was done to minimize risk of contamination of one group by the other.

Survey Instrument and Measures — The instrument used for the baseline measurement among the participants was in the form of a structured selfadministered questionnaire (five sections, 73 items), described elsewhere ${ }^{12,13)}$ (a copy of the questionnaire could be obtained from the corresponding author). Questions included participants' demographic characteristics (7 questions), knowledge of potential HIV risk situations (16 questions), attitudes towards prevention of HIV infection and people with AIDS (described as attitude towards AIDS) (4 questions), selfreported sexual behaviour (6 questions), and perception of risk (30 questions). Composite HIV/AIDS knowledge and attitude scores were generated based on the responses ranging from 'very high risk' to 'very low risk' to sixteen 6-point likert scale knowledge questions on different HIV/AIDS risk activities and four 5-point likert scale attitude questions ranging from 'strongly disagree' to 'strongly agree.' These had satisfactory ${ }^{14,15)}$ internal reliability as indicated by Cronbach's alpha $(\alpha)$ scores of above 0.70 in the baseline and follow-up surveys for both knowledge and attitude components.

Summary Score Calculation for Knowledge of HIV/AIDS, Attitudes towards HIV/AIDS and Perceptions of Risk — For the sixteen questions on the knowledge of HIV and AIDS, a summary score was developed, assigning 1 mark for correct answer and 0 mark for incorrect or uncertain answers. A maximum of 16 marks could be scored in the knowledge questions. For the four questions on the attitudes towards HIV/AIDS, five-point likert scale (don't know, strongly agree, agree, disagree \& strongly disagree) responses carried a mark of 0 to 4. A summary attitude score could then range between 0 and 16 marks. The higher score a subject obtained, the better attitudes the subject possessed. For the purpose of analyses, high score (score above the median) and low score (score below the median) were generated.

Respondents' perceptions of risk were assessed from their responses to 30 questions (a list could be obtained from the corresponding author) which included activities related to normal everyday life as well as activities involving high-risk behaviour. Respondents were asked to rate on a scale ranging from $0 \%$ (absolutely no chance) to $100 \%$ (certainly will 
happen) the probabilities of certain consequences occurring (e.g. being infected with sexually transmitted disease (STD)/HIV, developing heart disease or lung cancer, infected with hepatitis and so on). These 30 items were factor analysed with varimax rotation for the purpose of data reduction. Principal component analysis identified four factors, which accounted for $43 \%$ of the variance, and emphasised perceived STD risk (10 questions), perceived illness (7 questions), perceived accidental occurrence (5 questions) and sexual promiscuity (5 questions). Factor scores were computed by summing scores for individual items and used as dependent measures for perception of risk $(\alpha>0.75)$ in the baseline and follow-up studies. The higher the score the higher the perception of risk of the individual.

Intervention — The educational intervention of this study followed the information-motivationbehavioural-skills model. ${ }^{16)}$ According to this model, AIDS-risk-reduction information and motivation work largely through AIDS-risk-reduction behavioural skills to affect AIDS-risk-reduction behavioural change. We followed this model because this was reported to be easy to implement with high satisfaction rate among the participants. ${ }^{16)}$

One 90-min session educational intervention was provided among all the subjects in the intervention group at the same time, between January and August 1997. The intervention consisted of a 30-min presentation given by one of the authors (ASMA), which included definition of HIV and AIDS, modes of HIV transmission, common misconceptions about AIDS, risky behaviours and risk reduction through the practice of safer sex; reading of HIV/AIDS awareness and risk reduction pamphlets for $15 \mathrm{~min}$; and a 30-min show of a Chinese dialogue video tape entitled "AIDS and the Workplace," produced by a local AIDS organization to promote AIDS awareness and prevention methods. During the last $15 \mathrm{~min}$ participants were encouraged to ask questions related to the presentation, videotape or written materials.

The comparison group initially received no intervention, but after the measurement the intervention was repeated to comparison group subjects for ethical reasons. This study was approved by the Ethics Committee of the Faculty of Medicine of the University of Hong Kong, HKSAR, and performed in the HKSAR.

\section{Procedures -}

Baseline and Follow-Up Assessment: At baseline, all participants completed the self-admin- istered questionnaire (copies of the intervention measure and intervention protocol are available upon request).

Intervention Group: One week after the baseline study, the intervention group received the intervention while the comparison group received no intervention. After four months, the intervention group were surveyed again with a modified version of the baseline questionnaire containing additional questions on sexual behaviour during the period between the intervention and the current follow-up and intention to change behaviour (follow-up questionnaire).

Comparison Group: Four months after completing the baseline study, the comparisongroup were surveyed again using the follow-up questionnaire. The comparison group then received the intervention. The contents, length and format of the session were identical to that of the intervention provided to intervention group participants.

Data Processing and Analyses — Differences in responses between intervention and comparison groups at baseline and follow-up, and between groups at different points of time were evaluated by $\chi^{2}$ test with continuity correction. Non-parametric Wilcoxon Matched-Pairs Signed-Ranks Test was used to assess differences between the two groups over the period of time on composite scores of AIDS knowledge, attitudes towards AIDS, and the four factors of perceived risk. Since the distribution of scores in different variables was skewed, the 'median' was considered to be a more appropriate measure than the 'mean' and was used widely during the analysis.

\section{RESULTS}

Table 1 shows the baseline characteristics of participants. At baseline, intervention and comparisongroups did not differ with respect to demographic characteristics, sexual experience, median AIDS knowledge scores, median attitudes towards AIDS scores and HIV risk perceptions (four factors) scores.

\section{Intervention Effects}

During the intervention period (duration between baseline and follow-up at four-months) equal proportions $(6 \%)$ of intervention (4/67) and comparison group (3/51) respondents reported having had sex. Due to the low prevalence of sexual behaviour 
Table 1. Characteristics of the Study Population at Baseline: Intervention and Comparison Group

\begin{tabular}{|c|c|c|c|}
\hline & \multicolumn{3}{|c|}{ Baseline survey } \\
\hline & $\begin{array}{c}\text { Intervention } \\
(n=67)\end{array}$ & $\begin{array}{c}\text { Comparison } \\
\quad(n=51)\end{array}$ & $p$-value ${ }^{a)}$ \\
\hline & $n \quad(\%)$ & $n(\%)$ & \\
\hline \multicolumn{4}{|l|}{ Age } \\
\hline$<18$ years & $13(19.4)$ & $9(17.6)$ & $>0.05$ \\
\hline $18-25$ years & $52(77.6)$ & $40(78.4)$ & \\
\hline 26 or more & $2(3.0)$ & $2(4.0)$ & \\
\hline Mean length of stay in Hong Kon (years) & 19.53 & 19.58 & $>0.05$ \\
\hline \multicolumn{4}{|l|}{ Educational attainment } \\
\hline Secondary school or below & $58(86.6)$ & $45(88.2)$ & $>0.05$ \\
\hline College/university & $9(13.4)$ & $5(11.8)$ & \\
\hline \multicolumn{4}{|l|}{ Marital status } \\
\hline Married & $3(4.5)$ & $2(4.0)$ & $>0.05$ \\
\hline Never married & $64(95.5)$ & $48(96.0)$ & \\
\hline \multicolumn{4}{|l|}{ Religious belief } \\
\hline Yes & $11(16.4)$ & $8(15.7)$ & $>0.05$ \\
\hline None & $56(83.6)$ & $43(84.3)$ & \\
\hline \multicolumn{4}{|l|}{ Sexual experience during the previous 1 year ${ }^{b)}$} \\
\hline Yes & $9(13.6)$ & $7(13.7)$ & $>0.05$ \\
\hline No & $57(86.4)$ & $44(86.3)$ & \\
\hline \multicolumn{4}{|l|}{ Knowledge score } \\
\hline Above median $(>10)$ & $34(56.7)$ & $24(47.1)$ & $>0.05$ \\
\hline Below median $(<10)$ & $26(43.3)$ & $27(52.9)$ & \\
\hline \multicolumn{4}{|l|}{ Attitudes score } \\
\hline Above median $(>12)$ & $34(52.3)$ & $24(47.1)$ & $>0.05$ \\
\hline Below median $(<12)$ & $31(47.7)$ & $27(52.9)$ & \\
\hline \multicolumn{4}{|l|}{ Factor 1 (STD risk) } \\
\hline Above median $(>55)$ & $25(38.5)$ & $22(43.1)$ & $>0.05$ \\
\hline Below median $(<55)$ & $40(61.5)$ & $29(56.8)$ & \\
\hline \multicolumn{4}{|l|}{ Factor 2 (Illness) } \\
\hline Above median $(>39)$ & $36(53.7)$ & $27(52.9)$ & $>0.05$ \\
\hline Below median $(<39)$ & $31(46.3)$ & $24(47.1)$ & \\
\hline \multicolumn{4}{|l|}{ Factor 3 (accidental occurrence) } \\
\hline Above median $(>24)$ & $30(49.2)$ & $29(56.9)$ & $>0.05$ \\
\hline Below median $(<24)$ & $31(50.8)$ & $22(43.1)$ & \\
\hline \multicolumn{4}{|l|}{ Factor 4 (sexual promiscuity) } \\
\hline Above median $(>17)$ & $38(57.6)$ & $23(45.1)$ & $>0.05$ \\
\hline Below median $(<17)$ & $28(42.4)$ & $28(54.9)$ & \\
\hline \multicolumn{4}{|l|}{ Intention to use condoms with regular partners } \\
\hline Yes & $17(25)$ & $11(22)$ & $>0.05$ \\
\hline No & $50(75)$ & $40(78)$ & \\
\hline \multicolumn{4}{|c|}{ Intention to use condoms with irregular partners } \\
\hline Yes & $21(31)$ & $15(29)$ & $>0.05$ \\
\hline No & $46(69)$ & $36(71)$ & \\
\hline
\end{tabular}

a) $p$-value for $\chi^{2}$ test; $b$ ) only those who are sexually active.

among the participants we did not explore this further.

To assess the effects of intervention on AIDS knowledge, attitudes towards AIDS, and perception of risks, the composite scores of these variables in follow-up were compared with the scores in the baseline study for both intervention and comparison group respondents (Table 2). At follow-up, re- 
Table 2. AIDS Knowledge and Attitude Score, Self-Efficacy and Risk Perceptions among Respondents in the Intervention and Comparison Group

\begin{tabular}{|c|c|c|c|}
\hline \multirow[b]{2}{*}{ Scores } & \multicolumn{3}{|c|}{ Ranges of scores: baseline $v s$. follow-up at four months (intervention group) } \\
\hline & Baseline & Follow-up at four months & $p$-value ${ }^{a)}$ \\
\hline Knowledge (median) & $4-13 \quad(10)$ & $7-13(12)$ & 0.042 \\
\hline Attitudes (median) & $3-20 \quad(12)$ & $8-20(16)$ & 0.000 \\
\hline Perceived STD risk (median) & $18-93 \quad(55)$ & $15-97(66)$ & 0.005 \\
\hline Illness risk (median) & $12-61 \quad(39)$ & $7-65(37.5)$ & 0.503 \\
\hline Accidental occurrence risk (median) & $8-42 \quad(24)$ & $8-43(25)$ & 0.855 \\
\hline \multirow[t]{2}{*}{ Sexual promiscuity (median) } & $5-41 \quad(17)$ & $2-41(16)$ & 0.977 \\
\hline & \multicolumn{3}{|c|}{ Ranges of scores: baseline $v s$. follow-up at four months (comparison group) } \\
\hline Scores & Baseline & Follow-up at four months & $p$-value ${ }^{a)}$ \\
\hline Knowledge (median) & $6-13 \quad(10)$ & $6-13(10)$ & 0.367 \\
\hline Attitudes (median) & $4-18 \quad(10)$ & $10-20(13)$ & 0.000 \\
\hline Perceived STD risk (median) & $17-100(68.5)$ & $23-91(70)$ & 0.066 \\
\hline Illness risk (median) & $13-58 \quad(34.5)$ & $11-62(38)$ & 0.001 \\
\hline Accidental occurrence risk (median) & $7-41 \quad(25)$ & $9-41(24)$ & 0.179 \\
\hline Sexual promiscuity (median) & $5-58 \quad(13)$ & $2-48(10)$ & 0.152 \\
\hline
\end{tabular}

spondents who participated in the intervention tended to differ from the comparison group respondents in AIDS knowledge scores and in perceived STD risk scores; intervention group respondents had become more knowledgeable about AIDS risk and perceived themselves more at risk to STD infection than the comparison group. The AIDS attitude scores were significantly higher at follow-up for both the intervention and comparison group. Among comparison but not intervention group respondents, there was a significant difference in perceived risk of illness scores.

Improvements in the intention to use condom with regular and irregular partners were observed among respondents in both the intervention and comparison group at follow-up. At baseline, 25 and $31 \%$ of the intervention group respondents and 22 and $29 \%$ of the comparison group respondents (no significant difference) intended using condoms with regular and occasional partners respectively. However at follow-up, a significantly higher proportion of intervention group than comparison group respondents intended to use condoms with regular (55 vs. $\left.39 \% ; \chi^{2}=5.28, \mathrm{df}=1, p<0.002\right)$ and irregular $(72$ vs. $\left.41 \% ; \chi^{2}=4.68, \mathrm{df}=1, p<0.001\right)$ partners.

\section{Behaviour Change}

At follow-up, 43/62 (69\%) of the intervention group and 33/51 (65\%) of the comparison group respondents reported that they had changed their behaviour in general as a result of their understand- ing about HIV/AIDS. The frequency of discussions about AIDS with partners or friends and their determination of using condoms with a new partner were not significantly different between intervention and comparison group participants. A significantly higher proportion of intervention (33\%) than comparison $(13 \%)$ group respondents had talked about condom use for safer sex with friends $\left(\chi^{2}=4.48, \mathrm{df}=1\right.$, $p=0.034)$.

\section{DISCUSSION}

This is the first study to assess the effectiveness of an AIDS educational intervention amongst the Chinese youths who are growing up in a culturally sensitive society. Although, there are limitations this study is useful as this generated new information, which is not yet documented among the population who has received very little HIV prevention information. The findings suggest that one 90-min session educational intervention that was offered to a culturally conservative young Chinese in a training setting is effective. The intervention program was not effective in improving all the measures and few unanticipated findings for the comparison group emerged. Relative to the comparison group, intervention group had improved in two of the six measurement components (comparison group in one component), an improved intention to use condoms with regular and irregular partners and more discus- 
sion with friends about safer sex. It appears that this short intervention program, which is practicable in the real world situation (because many adolescents would not attend multiple sessions), would be useful if this could be implemented. There are, however, a number of limitations, which needs to be considered while evaluating the findings. These are small sample size, low participation rate, risk of contamination of the control group with the intervention group, and short time of follow up.

The significant improvement in knowledge is in line with other international studies ${ }^{17,18)}$ and demonstrates the need to disseminate information to replace myths generated by denial and ignorance. A significant increase in attitudes towards AIDS among subjects in both groups suggests that both groups have obtained information from similar sources such as media or other publicity materials. Also, the baseline measure itself may have encouraged participants with less HIV/AIDS awareness to educate them. It was also possible that the intervention group contaminated the comparison group, because both groups lived in the same campus and met during social gatherings or other activities. This would have provided them with the opportunities to share information on HIV/AIDS.

Perception of risk from certain activities is associated with the intention of being involved in such activities. ${ }^{19)}$ In general people tend to be more optimistic about future adverse life events, ${ }^{20)}$ especially long-term ones such as the outcomes of high-risk sexual behaviour. This is associated with low risk perception. ${ }^{21)}$ In this study, the perception of STD risk among respondents in the intervention group increased due to their participation in the intervention. The personal experience of any illness might have facilitated to the perception that preventive measures are effective ways to minimise illness risk, resulting in a higher perceived illness risk among comparison group respondents at follow-up.

The higher intention to use condoms with both regular and irregular partners among respondents in the comparison group suggests one of two things. First, there is the possibility of a social desirability response set. The limitations on intentions translating into behaviour are well known and are a potentially important source of error in these data. Second, that the intervention has changed their attitudes towards condom use. This is encouraging because behavioural intentions to perform safer sex are reasonable predictors of actual behaviour change. ${ }^{17)}$

The educational programme in this study only included one session, which was not enough to cover all the issues regarding HIV/AIDS. Also the 90-min duration was tiring for participants. We tried to arrange several short sessions covering different aspects of HIV/AIDS preventive education, which was suggested to be most effective, ${ }^{22}$ but were not permitted by the authority. The impression was that even though the intervention was more effective in the intensive manner, this would not be practical to implement at the school. Other studies discussed the ineffectiveness of 'AIDS education alone' as an intervention, and suggested the need to include other factors such as cognitive attitudinal factors, risk reduction skills training and problem solving support within the intervention framework. ${ }^{23)}$

The results of this study identified the effectiveness and limitations of an HIV/AIDS educational programme targeting Chinese young adults. More importantly this is the first intervention study targeted towards young people in a culture where sexuality is not discussed. Thus the study may serve as an initial attempt in conducting similar studies in the future with new theory-driven intervention program in Hong Kong and the Peoples' Republic of China, which share the same cultural sensitivity. Any future study should examine the incremental effects of a long-term follow-up, which was found in other samples to be an important measure to maintain the stability of programme effects over time ${ }^{15)}$ and whether intervention of the type used in this study would be effective among Chinese youths with higher rate of sexual activity. Considering the limitations identified in this study, future studies are also expected to be theoretically more rigorous.

Acknowledgements We thank Mr. S. Ma and Dr. C. M. Wong of the Department of Community Medicine at the University of Hong Kong (HKU) for their assistance during the statistical analysis and Ms. J. H. Zhong of the Research Services Section at HKU for her editorial assistance.

\section{REFERENCES}

1) UNAIDS (Joint United Nations Programme on AIDS)/WHO (World Health Organisation) (2000) AIDS epidemic update, December, World Health Organisation, Geneva, Switzerland.

2) UNAIDS/WHO (1998) Report on the global HIV/ AIDS epidemic, June, World Health Organisation, Geneva, Switzerland. 
3) Rosenthal, D., Moore, S. and Buzwell, S. (1994) Homeless youths: sexual and drug-related behaviour, sexual beliefs and HIV/AIDS risk. AIDS Care, 6, 1 .

4) Census \& Statistics Department (1996) Report of the 1996 Population By-census, Hong Kong Government, Hong Kong.

5) Department of Health, Government of the Hong Kong SAR (1999) Hong Kong STD/AIDS Update, vol. 5, no. 1-4, http://www.info.gov.hk/aids.

6) AIDS prevention and care committee (APCC) of the Hong Kong Advisory Council on AIDS (2000) HIV prevention and care in youth: principles of strategy, Hong Kong Government, Hong Kong, http// www.info.gov.hk/aids, pp. 1-23.

7) Family Planning Association of Hong Kong (1991) Working report on: Youth sexuality study (out of school youth survey) 1991, Family Planning Association of the Department of Health, Hong Kong, pp. 10-29.

8) Family Planning Association of Hong Kong (1996) Working report on: Youth sexuality study among inschool and out of school youth-1996.

9) EPI Centre (1996) Evaluation of health promotion and social interventions. In Review of effectiveness of sexual health promotion interventions for young people. Social Science Research Unit, London University Institute of Education, London.

10) Cheng, K. T., Chan, Y. F. and Chau, K. V., et al. (1993) What do adolescents in Hong Kong know and think about AIDS and sex? Hong Kong Practitioner, 15, 12-18.

11) Davis, C. and Tang, C. S. (1998) HIV and knowledge among high-risk Chinese adolescents in Hong Kong: implications of a prevention programme. $J$. of HIV/AIDS prevention \& education for adolescents \& children, 2, 55-66.

12) Abdullah, A. S. M., Hedley, A. J. and Fielding, R. (1999a) High risk sexual behaviour in travellers. Lancet, 353, 595.

13) Abdullah, A. S. M., Fielding, R., Hedley, A. J. and Ma, S. (1999b) Training medical students about
HIV/AIDS. Med. Educ., 33, 472-473.

14) Ashworth, C. S., DuRant, R. H., Gaillard, G. and Rountree, J. (1994) An experimental evaluation of an AIDS educational intervention for WIC mothers. AIDS Educ. Prev., 6, 154-162.

15) DiClemente, R. J. (1991) Predictors of HIV preventive sexual behaviour in a high-risk adolescent population: The influence of perveived peer norms and sexual communication on increased adolescentsconsistent use of condoms. J. Adolesc. Health, 12, 385-390.

16) Fisher, J. D. and Fisher, W. A (1992) Changing AIDS risk behaviour. Psychol. Bull., 111, 455-474.

17) Shulkin, J. J., Mayer, J. A., Wessel, L. G. and Moor, C. D., et al. (1991) Al. Effects of a peer-led AIDS intervention with university students. Journal of American College Health 1991, 40, 75-79.

18) Mbizvo, M. T., Kasule, J., Gupta, V. and Rusakaniko, S., et al. (1997) Effects of a randomised health education intervention on aspects of reproductive health knowledge and reported behaviour among adolescents in Zimbabwe. Soc. Sci. Med., 44, 573-577.

19) Rosenstock, I. M., Strecher, V. J. and Becker, M. H. (1988) Social learning theory and the health belief model. Health Educ. Q, 15, 175-183.

20) Weinstein, N. D. (1980) Unrealistic optimism about future life events. J. Pers. Soc. Psychol., 39, 806820.

21) Abdullah, A. S. M., Hedley, A. J. and Fielding, R. (1998) Travel, sexualbehaviour and risk to HIV infection. Hong Kong Medical Association Journal, 4, 137-144.

22) Rotheram-Borus, M. J., Koopman, C., Haignere, C. and Davies, M. (1991) Reducing HIV sexual risk behaviours among runaway adolescents. JAMA, 266, 1237-1241.

23) Kelly, J. A., Murphy, D. A., Washington, C. D. and Wilson, T. S., et al. (1994) The effects of HIV/AIDS intervention groups for high risk women in urban clinics. American journal of Public Health, 84, 1918-1922. 